Proc. Indian Acad. Sci. (Chem. Sci.), Vol. 102, N0. 5, October 1990, pp. 629-634.

(C) Printed in India

\title{
Raman scattering in magnetic materials
}

\author{
R A YADAV \\ Department of Physics, Banaras Hindu University, Varanasi 221005, India
}

\begin{abstract}
The new selection rules formulated for gyrotropic materials in presence of the Faraday Rotation by earlier workers are critically examined in the light of the work of Slobodyanyuk and Schaack. It is argued that it is difficult to distinguish between the two effects, namely, the position dependence (size effect) and phase dependence (interference effect) causing intensity anomalies in Raman scattering.
\end{abstract}

Keywords. Raman scattering; magnetic materials; size effect; interference effect; intensity anomalies.

\section{Introduction}

Owing to their wide ranging applications magnetic materials have been a subject of extensive studies for the past two decades. Studies on the morphic effects in magnetic materials are of prime importance in elucidating their structural properties. Changes in the Raman and IR activity of the $q \approx o$ optical phonons and the splittings of the phonon degeneracies in crystals due to externally applied axial (magnetic) and polar (electric) forces are referred to as morphic effects. A series of papers on this subject has been published by Burstein and co-workers (see Anastassakis and Burstein 1973). The Raman and IR activity of an optical phonon essentially depends on the crystal symmetry. Therefore, the changes in the Raman and IR activity of an optical phonon demand an essential lowering of the crystal symmetry. Such a lowering is well-known to occur when magnetic materials undergo a temperature-caused phase transition from the paramagnetic to the magnetic state due to breakdown of the time reversal symmetry of the crystal (Anastassakis and Burstein 1973).

The present work is concerned with the Raman scattering in magnetic materials in the presence of a magnetic field. The lowering of the crystal symmetry would result in the observation of more Raman frequencies as compared to the usual symmetryallowed case. Song et al (1973) have studied the Raman spectra of iron garnets and have observed a number of additional Raman frequencies. However, they have summarized their work without mentioning any conclusive origin of the additional Raman frequencies. Peng-Xiang and An (1983) have traced the origin of these additional frequencies to the effect of Faraday rotation on the phonon Raman scattering. On the other hand Slobodyanyuk and Schaack (1987) have criticized the work of Peng-Xiang and An (1983) and attribute the additional frequencies to the interference effects between the two orthogonally polarised normal modes of electromagnetic radiation propagating in the anisotropic crystal either in the incident or in the scattered beam or in both. In the present work the formalism of Peng-Xiang and 
An (1983) has been critically reviewed in the light of the points raised by Slobodyanyuk and Schaack (1987).

\section{Theory}

Let $I_{i}$ and $I_{s}$ be the intensities of the incident and scattered radiations and $\hat{e}_{i}$ and $\hat{e}_{s}$ be the electric field unit vectors of the incident and scattered radiation and $\vec{X}$ be the Raman tensor of the excitation under consideration. Then the scattered intensity $I_{s}$ is given by

$$
I_{s}=C_{0} I_{i}\left|\hat{e}_{s} \vec{X} \hat{e}_{i}\right|^{2},
$$

where $C_{0}$ is a constant related to the frequencies of the incident and scattered radiations and the power spectrum of the excitation involved. As magnetic materials usually have strong absorption it is desirable to study the Raman spectra in a back-scattering arrangement. Let the incident and scattered radiations travel along the $\pm Z$ axis and the material occupy $Z>0$ space. Then the incident intensity at a position $Z$ is given by,

$$
I_{i}(Z)=I_{i}(0) e^{-\alpha Z},
$$

where $I_{i}(0)$ is the intensity of the incident radiation at the material surface and $\alpha$ is the material absorption coefficient. In the presence of the magnetic field Faraday rotation takes place and the polarization of the incident radiation at the position $Z$ is given by

$$
\hat{e}_{i}(Z)=\overleftrightarrow{T}_{F} \hat{e}_{i}(0)
$$

where $\hat{e}_{i}(Z)$ and $\hat{e}_{i}(0)$ are the electric field unit vectors at a position $Z$ and the material surface respectively, and $\widetilde{T}_{F}$ is the transformation tensor which rotates the electric field vector to a direction $\theta$ given by

$$
\theta=\theta_{F} Z,
$$

where $\theta_{F}$ is the specific Faraday rotation defined as the angle of rotation of the material of unit length. The total intensity of the back-scattered radiation is given by

$$
I_{s}=\int_{0}^{\infty} C_{0} I_{i}(0)\left|\hat{e}_{s}(0) \overleftrightarrow{T}_{F} \overleftrightarrow{X} \overleftrightarrow{T}_{F} \hat{e}_{i}(0)\right|^{2} e^{-2 \alpha Z} \mathrm{~d} Z,
$$

where $\hat{e}_{s}(Z)$ is expressed by a relation similar to (3).

The exponential $e^{-2 \alpha Z}$ in (5) appears due to the fact that the radiation travels twice the distance $Z$ in the back-scattering arrangement. The symbols $\hat{e}_{i}(0), \hat{e}_{s}(0), T_{F}$ and $\vec{X}$ have the following matrix forms:

$$
\begin{aligned}
& \hat{e}_{i}(0)=\left(\begin{array}{c}
\alpha_{1} \\
\alpha_{2} \\
0
\end{array}\right), \quad \hat{e}_{s}(0)=\left(\beta_{1} \beta_{2} 0\right), \\
& \vec{T}_{F}=\left(\begin{array}{ccc}
\cos \theta & \sin \theta & 0 \\
-\sin \theta & \cos \theta & 0 \\
0 & 0 & 1
\end{array}\right), \quad \vec{X}=\left(\begin{array}{lll}
a & b & e \\
c & d & g \\
f & h & i
\end{array}\right),
\end{aligned}
$$


On introducing these expressions in (5) and integrating it, the following expression is obtained,

$$
\begin{array}{r}
I_{s}=\frac{C_{0} I_{i}(0)}{2 \alpha}\left[\frac{A^{2}+B^{2}+2 C^{2}}{2}+\frac{B^{2}-A^{2}}{2\left(1+16 Q^{2}\right)}+\frac{2 B C}{\left(1+4 Q^{2}\right)}+\right. \\
\left.+\frac{4 Q C A}{\left(1+4 Q^{2}\right)}+\frac{4 Q A B}{\left(1+16 Q^{2}\right)}\right]
\end{array}
$$

where $Q=\theta_{F} / 2 \alpha$ is called figure of merit and is a characteristic property of the material and $A, B$ and $C$ are constants given by

$$
\begin{aligned}
& A=\frac{1}{2}\left[a\left(\alpha_{2} \beta_{1}-\alpha_{1} \beta_{2}\right)-b\left(\alpha_{1} \beta_{1}+\alpha_{2} \beta_{2}\right)+c\left(\alpha_{1} \beta_{1}+\alpha_{2} \beta_{2}\right)+d\left(\alpha_{2} \beta_{1}-\alpha_{1} \beta_{2}\right)\right] \\
& B=\frac{1}{2}\left[a\left(\alpha_{1} \beta_{1}+\alpha_{2} \beta_{2}\right)+b\left(\alpha_{2} \beta_{1}-\alpha_{1} \beta_{2}\right)+c\left(\alpha_{1} \beta_{2}-\alpha_{2} \beta_{1}\right)+d\left(\alpha_{2} \beta_{2}+\alpha_{1} \beta_{1}\right)\right] \\
& C=\frac{1}{2}\left[a\left(\alpha_{1} \beta_{1}-\alpha_{2} \beta_{2}\right)+b\left(\alpha_{2} \beta_{1}+\alpha_{1} \beta_{2}\right)+c\left(\alpha_{1} \beta_{2}+\alpha_{2} \beta_{1}\right)+d\left(\alpha_{2} \beta_{2}-\alpha_{1} \beta_{1}\right)\right]
\end{aligned}
$$

In the absence of Faraday rotation $Q=0$ and (6) simplifies to

$$
I_{s}^{\prime}=\frac{C_{0} I_{i}(0)}{2 \alpha}(B+C)^{2}
$$

For non-absorbing crystals, $\alpha=0$ and therefore, $Q$ is no longer a well-defined property of the material. Under such conditions the intensity of the scattered radiation is given by,

$$
\begin{aligned}
I_{s}=C_{0} I_{i}(0) & {\left[U^{2} \int \cos ^{4}\left(\theta_{F} Z\right) \mathrm{d} Z+V^{2} \int \sin ^{4}\left(\theta_{F} Z\right) \mathrm{d} Z+\right.} \\
& +W^{2} \int \sin ^{2}\left(\theta_{F} Z\right) \cos ^{2}\left(\theta_{F} Z\right) \mathrm{d} Z+2 U V \int \sin ^{2}\left(\theta_{F} Z\right) \cos ^{2}\left(\theta_{F} Z\right) \mathrm{d} Z+ \\
& \left.+2 V W \int \sin ^{3}\left(\theta_{F} Z\right) \cos \left(\theta_{F} Z\right) \mathrm{d} Z+2 U W \int \sin \left(\theta_{F} Z\right) \cos ^{3}\left(\theta_{F} Z\right) \mathrm{d} Z\right]
\end{aligned}
$$

where, $U=B+C, V=C-B$ and $W=2 A$.

Now, assuming finite thickness $(L)$ of the sample along the $Z$ direction (9) becomes

$$
\begin{aligned}
I_{s}= & \frac{C_{0} I_{i}(0)}{\theta_{F}}\left[U^{2}\left(\frac{12 \theta_{F} L+8 \sin 2 \theta_{F} L+\sin 4 \theta_{F} L}{32}\right)+\right. \\
& +V^{2}\left(\frac{12 \theta_{F} L-8 \sin 2 \theta_{F} L+\sin 4 \theta_{F} L}{32}\right)+\left(2 U V+W^{2}\right) \times \\
& \left.\times\left(\frac{4 \theta_{F} L-\sin 4 \theta_{F} L}{32}\right)+\frac{2 V W \sin ^{4} \theta_{F} L}{4}+\frac{2 U W\left(1-\cos ^{4} \theta_{F} L\right)}{4}\right]
\end{aligned}
$$


Now for $\theta_{F} L=2 n \pi$ where $n$ is an integer (10) gives

$$
I_{s}=C_{0} l_{i}(0) L\left[C^{2}+\frac{1}{2}\left(A^{2}+B^{2}\right)\right]
$$

Further, in the absence of the Faraday rotation, $\left(\theta_{F}=0\right),(10)$ gives the scattered intensity as zero. It is quite understandable because the only mechanism for back-scattering in this formulation is the Faraday rotation.

Thus, from (6) and (9) it is clear that the intensity of the scattered radiation depends on $\theta_{F}$ irrespective of whether the material is absorbing or not.

\section{Example}

The importance of the above formalism may be realised by taking the example of the iron garnet $-\mathrm{YIG}\left(\mathrm{Y}_{3} \mathrm{Fe}_{5} \mathrm{O}_{12}\right)$ crystal which has the space group $O_{h}^{10}$ and calculating $I_{s}$ [from (6)] and $I_{s}^{\prime}[$ from (8)]. Using Raman tensors $(\vec{X})$ for the space group $O_{h}^{10}$ as,

Species

$$
\begin{gathered}
A_{1 g} \\
\vec{X}\left(\begin{array}{lll}
a & 0 & 0 \\
0 & a & 0 \\
0 & 0 & a
\end{array}\right),\left(\begin{array}{ccc}
b & 0 & 0 \\
0 & -b & 0 \\
0 & 0 & 0
\end{array}\right), \frac{1}{\sqrt{3}}\left(\begin{array}{ccc}
b & 0 & 0 \\
0 & b & 0 \\
0 & 0 & -2 b
\end{array}\right), \\
T_{2 g}^{1} \\
\left(\begin{array}{lll}
0 & d & 0 \\
d & 0 & 0 \\
0 & 0 & 0
\end{array}\right),\left(\begin{array}{lll}
0 & 0 & d \\
0 & 0 & 0 \\
d & 0 & 0
\end{array}\right),\left(\begin{array}{lll}
0 & 0 & 0 \\
0 & 0 & d \\
0 & d & 0
\end{array}\right)
\end{gathered}
$$

and the $\hat{e}_{i}(0)$ and $\hat{e}_{s}(0)$ for the five configurations as,

$$
\begin{array}{lcc}
\text { Configuration } & \hat{e}_{i}(0) & \hat{e}_{s}(0) \\
\langle 100\rangle_{\perp} & \langle 100\rangle & \langle 010\rangle \\
& \alpha_{1}=1, \alpha_{2}=0 & \beta_{1}=0, \beta_{2}=1 \\
\langle 100\rangle_{\|} & \langle 100\rangle & \langle 100\rangle \\
& \alpha_{1}=1, \alpha_{2}=0 & \beta_{1}=1, \beta_{2}=0 \\
\langle 110\rangle_{\perp} & \langle 110\rangle & \langle 110\rangle \\
& \alpha_{1}=1 / \sqrt{2}, \alpha_{2}=-1 / \sqrt{2} & \beta_{1}=1 / \sqrt{2}, \beta_{2}=1 / \sqrt{2} \\
\langle 110\rangle & \langle 110\rangle & \langle 110\rangle \\
& \alpha_{1}=1 / \sqrt{2}, \alpha_{2}=-1 / \sqrt{2} & \beta_{1}=1 / \sqrt{2}, \beta_{2}=-1 / \sqrt{2} \\
\langle 100\rangle & \langle 100\rangle & \langle 110\rangle
\end{array}
$$

the intensities $I_{s}^{\prime}$ and $I_{s}$ are calculated as given in table 1 .

It can be seen from table 1 that the phonons belonging to the species $A_{1 g}$ and $E_{g}^{2}$ are Raman-inactive in the absence of the Faraday rotation and become Raman-active in the presence of the Faraday rotation. We now make the following observations: 
Table 1. Calculated intensities.

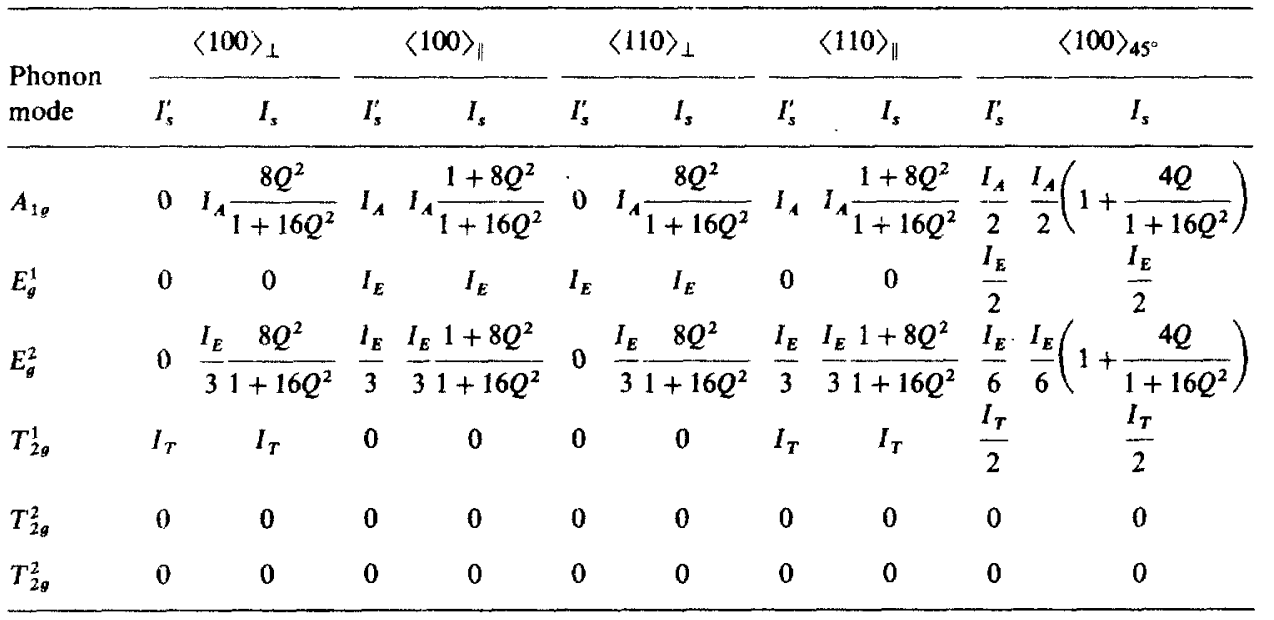

where $I_{A}=\frac{C_{0} I_{i}(0) a^{2}}{2 \alpha}, I_{E}=\frac{C_{0} I_{i}(0) b^{2}}{2 \alpha}, I_{T}=\frac{C_{0} I_{i}(0) d^{2}}{2 \alpha}$.

(1) The Raman tensor of the species $E_{g}^{1}$ (Peng-Xiang and An 1983) should be replaced by

$$
\left(\begin{array}{rrr}
b & 0 & 0 \\
0 & -b & 0 \\
0 & 0 & 0
\end{array}\right)
$$

for example (see Hurrell et al 1968).

(2) Even with this correction of the Raman tensor, intensities $I_{s}$ and $I_{s}^{\prime}$ (Peng-Xiang and An 1983) could be reproduced only for two orientations $\langle 100\rangle_{\perp}$ and $\langle 100\rangle_{\|}$for all the six species in the present case as given in table 1 . However, for the remaining configurations $\langle 110\rangle_{1},\langle 110\rangle_{\|}$and $\langle 100\rangle_{45^{\circ}}$ intensities in the present case agree with those given (Peng-Xiang and An 1983) for the species $A_{1 g}, E_{g}^{1}, T_{2 g}^{1}, T_{2 g}^{2}$, and $T_{2 g}^{3}$. For $E_{g}^{2}$ species a factor of $1 / 3$ seems missing in Peng-Xiang and An (1983).

(3) It has been recently argued (Slobodyanyuk and Schaak 1987) that rather than seeking interpretation of the experimental results in terms of the position-dependent quantities, it is the polarisation property of the electromagnetic wave and its phase changes in a gyrotropic crystal which are responsible for the observed intensity anomalies. This alternative interpretation could be interesting if a detailed experiment on the effect of temperature on the selection rules using polarised electromagnetic waves is performed. It is not easy to distinguish the phenomena in which the scattered intensity is directly position-dependent (Peng-Xiang and An 1983) or occurs due to the interference of different electromagnetic waves (Slobodyanyuk and Schaak 1987). (4) The use of a single absorption coefficient assumed by Peng-Xiang and An (1983) for the linearly polarised light subjected to a Faraday rotation is not correct, according to Slobodyanyak and Schaak (1987). However, since the absorption coefficient varies fairly smoothly in the wavelength range covered by the phonon modes, using a single absorption coefficient in such a wavelength range would not introduce much error. 
Even if some error is introduced it would certainly not affect the selection rules worked out in Peng-Xiang and An (1983).

(5) A proper analysis for this problem, where the size of the sample could be arbitrarily small and the general (spin) polarization property of electromagnetic waves is taken into account, necessarily demands quantum field theoretic treatment. Obviously the use of the transformation tensor matrix and the macroscopic value of absorption coefficient are invalid in this case.

\section{Acknowledgement}

The author records his indebtedness to Dr S C Tiwari for getting him interested in this field.

\section{References}

Anastassakis E and Burstein E 1973 J. Phys. C5 2468

Hurrell J P, Porto S P S, Chang I F, Mitra S S and Bauman R P 1968 Phys. Rev. 173851

Peng-Xiang Z and An T 1983 J. Raman Spectrosc. 14326

Song J J, Klein P B, Wadsack R L, Selders M, Mroczkowski S and Change R K 1973 J.Opt. Soc. Am. 631135

Slobodyanyuk A V and Schaack G 1987 J. Raman Spectrosc, 18561 